\title{
99m Tc-NTP 15-5 Imaging for Cartilage Involvement in Experimental Rheumatoid Arthritis: Comparison with Routinely Used Molecular Imaging Methods and Sensitivity to Chronic Nonsteroidal Antiinflammatory Drug Treatment
}

\author{
Amit Khairnar ${ }^{1-4}$, Fabien Marchand ${ }^{3,4}$, Aurélien Vidal ${ }^{1,2}$, Monique Etienne ${ }^{3,4}$, Imen Miladi ${ }^{1,2}$, Philippe Auzeloux ${ }^{1,2}$, \\ Florent Cachin ${ }^{1,2,5}$, Alain Eschalier ${ }^{3,4}$, Jean-Michel Chezal ${ }^{1,2}$, Denis Ardid ${ }^{3,4}$, and Elisabeth Miot-Noirault ${ }^{1,2}$ \\ ${ }^{I}$ Clermont Université, Université d'Auvergne, Imagerie Moléculaire et Thérapie Vectorisée, BP 10448, Clermont-Ferrand, France; \\ ${ }^{2}$ INSERM, U 990, Clermont-Ferrand, France; ${ }^{3}$ Clermont Université, Université d'Auvergne, Pharmacologie Fondamentale et \\ Clinique de la Douleur, Clermont-Ferrand, France; ${ }^{4}$ INSERM U1107 NEURO-DOL, Clermont-Ferrand, France; and ${ }^{5}$ CLCC Jean \\ Perrin, Clermont-Ferrand, France
}

This study determined, using the intraarticular complete Freund

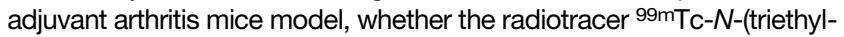
ammonium)-3-propyl-[15]ane-N5 (99mTc-NTP 15-5) targeting proteoglycans has a pathophysiologic validity for in vivo imaging of rheumatoid arthritis (RA) and its response to chronic nonsteroidal antiinflammatory drugs. Methods: We investigated the time course of cartilage remodeling by ${ }^{99 m T c-N T P} 15-5$ scintigraphy, bone damages by ${ }^{99 m T c}$-hydroxymethylene diphosphonate imaging, inflammation by ${ }^{18} \mathrm{~F}-\mathrm{FDG} \mathrm{PET}$, and joint proteoglycan content and pain behavior in animals, without and with meloxicam treatment. Paw circumference, thermal pain behavior, and histology as well as proteoglycan content of the whole joint were determined. Results: ${ }^{99 m}$ Tc-NTP $15-5$ showed specific tracer accumulation within RA joints, with a significant increase in scintigraphic ratio observed in RA versus shams from day 3 to day 28. ${ }^{18} \mathrm{~F}-\mathrm{FDG}$ evidenced uptake in RA joints from day 15 to day 29. Animals treated with meloxicam $(5 \mathrm{mg} / \mathrm{kg}$ ) exhibited a dose-dependent decrease in both ${ }^{99 m}$ Tc-NTP $15-5$ and ${ }^{18} \mathrm{~F}-\mathrm{FDG}$ uptake ratios versus saline-treated animals. $99 \mathrm{mTc}$-hydroxymethylene diphosphonate bone scans were only positive at day 14 in RA versus shams, with a significant effect of meloxicam. An increase in proteoglycans of RA joint and thermal pain behavior were observed and were dose-dependently reduced by meloxicam. Conclusion: These experimental results bring data in favor of the 99mTc-NTP 15-5 radiotracer for assessing, in vivo, cartilage remodeling in RA that could be used to monitor therapy.

Key Words: proteoglycans; rheumatoid arthritis; scintigraphy; 99mTc-NTP 15-5

J Nucl Med 2015; 56:798-804

DOI: $10.2967 /$ jnumed.114.151415

$\mathbf{R}$ heumatoid arthritis (RA) is a chronic, inflammatory, systemic autoimmune disease, which occurs in $1 \%$ of the population worldwide, affecting primarily the hands and feet, with a higher

Received Nov. 12, 2014; revision accepted Feb. 10, 2015.

For correspondence or reprints contact: Elisabeth Miot-Noirault, UMR 990 INSERM/UdA, 28 Place Henri Dunant, 63000 Clermont-Ferrand, France.

E-mail: elisabeth.noirault@inserm.fr

Published online Apr. 3, 2015.

COPYRIGHT (C) 2015 by the Society of Nuclear Medicine and Molecular Imaging, Inc. prevalence for women $(1,2)$. RA is characterized by severe synovial inflammation that results in pannus formation and progressive destruction of articular cartilage and bone, ultimately leading to stiffness, swelling, joint disability, and pain $(3,4)$. With the advent of disease-modifying antirheumatic drugs alone or in combinations with TNF- $\alpha$ inhibitors or nonsteroidal antiinflammatory drugs, RA remission has become a realistic goal but seems only possible if it is diagnosed at early stages (5-7). With such treatments emerging, in vivo assessment of RA has become a real need, for both an earlier diagnosis and an accurate monitoring of therapy efficacy. RA pathophysiology is not entirely understood, and no gold standard exists. Current imaging methods such as radiography, MR imaging, and ultrasound are all based on the detection of morphologic rather than biochemical changes in joints (6-9). Because molecular or cellular events often occur before any signs of structural or evident anatomic changes, authors consider that the key approach to the early diagnosis and monitoring of RA would be to detect these pathologic processes in vivo. In RA, the monitoring of inflammatory and destructive pathways of joint would undoubtedly be helpful for both basic research in arthritis and clinics. The clinical impact of cartilage in joint destruction was recently demonstrated to be much more relevant than previously considered, suggesting that more functional diagnostic and therapeutic effort should be spent on cartilage remodeling in RA (10-13).

In this context, in vivo molecular imaging of cartilage alteration and remodeling could help to develop more efficient diagnostic tools for the early staging of RA, disease monitoring, and diseasemodifying antirheumatic drug efficacy assessment.

The recent application of radionuclide imaging for preclinical imaging has opened access to many human pathophysiologic models at the molecular level, with a high sensitivity and a selectivity that can be enhanced by vectors.

For molecular imaging of joint, our strategy consisted of using the quaternary ammonium function, which exhibits a high affinity for proteoglycans, as a selective carrier of oxotechnetium complex to cartilaginous tissues. To that end, $\mathrm{N}$-(triethylammonium)-3propyl-[15]ane-N5 radiolabeled with ${ }^{99 \mathrm{~m}} \mathrm{Tc}$ was demonstrated to allow joint functional imaging in several experimental models of articular pathology and to specifically bind to human articular cartilage after ex vivo incubation (14-18). 
In this study, we determined the relevance and sensitivity of ${ }^{99 m}$ Tc- $N$-(triethylammonium)-3-propyl-[15]ane-N5 (99mTc-NTP 15-5) imaging for assessing cartilage involvement in a preclinical monoarthritis model, without and with meloxicam treatment. ${ }^{99 \mathrm{~m}}$ Tc-NTP $15-5$ cartilage imaging was compared with ${ }^{99 \mathrm{~m}} \mathrm{Tc}-$ hydroxymethylene diphosphonate ( $\left.{ }^{99 \mathrm{~m}} \mathrm{Tc}-\mathrm{HMDP}\right)$ bone imaging and ${ }^{18}$ F-FDG PET of inflammation. Paw circumference and thermal hypersensitivity associated with RA as well as joint histology and proteoglycans of whole-joint extracts were determined.

\section{MATERIALS AND METHODS}

\section{Experimental Model of Adjuvant Monoarthritis and Meloxicam Treatment}

Protocols were performed in accordance with the 2010/63/UE European Directive under the authorization of the French Directorate of Veterinary Services (authorization C63-113-10). Experiments were conducted on 40 animals randomly divided into 4 groups (3 groups induced for RA and shams). Each group consisted of 5-8 animals for longitudinal monitoring over the study and 4 additional animals included for proteoglycans dosage at days 4, 14, and 28 .

For RA induction, $12 \mu \mathrm{L}$ of complete Freund adjuvant (0640; DIFCO Laboratories) containing heat-inactivated Mycobacterium butyricum $(5 \mathrm{mg} / \mathrm{mL})$ in paraffin oil were intraarticularly injected into the tibiotarsal joint of the right hind paw of CD1 male mice (Charles River) on day $0(19,20)$. Shams received only the vehicle.

After RA induction, animals were treated with meloxicam (1 or $5 \mathrm{mg} / \mathrm{kg}$, subcutaneously) once day, from day 1 to day 29. All groups were then examined for both clinical, nociceptive criteria and functional imaging.

\section{Clinical Assessment of Monoarthritis by Paw Circumference} and Immersion Test

For each animal, the joint circumference of the pathologic and contralateral paws was determined at days 3,14 , and 28 . Results were expressed as the difference between the pathologic and the contralateral joint and averaged per group.

For the joint/paw immersion assay, water temperature was kept constant at $47.0 \pm 0.2^{\circ} \mathrm{C}$. Mice were maintained under a soft cloth except for their tail or hind paws, which were immersed in water until withdrawal (cutoff time of $30 \mathrm{~s}$ to minimize any potential damage). Paw withdrawal latency was assessed for each mouse at days 3, 7, 14, and 28. The test was performed $30 \mathrm{~min}$ after treatment, with the experimenter being masked to treatment.

\section{In Vivo Functional Imaging}

For all imaging procedures, ketamine (Imalgène 500; Rhone Mérieux) and xylazine (Rompun 2\%; Bayer) (4:1 ratio) were used to anesthetize animals.

For each radiotracer, the injected dose to each animal was determined by acquiring an image of the syringe before and after injection and counting (Capintec activimeter).

${ }^{18}$ F-FDG Imaging of Inflammation. ${ }^{18} \mathrm{~F}-\mathrm{FDG}$ imaging was performed using GLUCOTEP (Cyclopharma Laboratories). Each animal underwent 3 PET examinations at days 4, 15, and 29. Whole-body PET scans (30-min duration, 2 bed positions) were acquired $1 \mathrm{~h}$ after injection of ${ }^{18} \mathrm{~F}-\mathrm{FDG}$ (12 MBq/animal) using a small-animal PET device (eXplore VISTA; GE Healthcare), 250- to 700-keV energy window, and 6-ns coincidence time window. Image reconstruction used a 2-dimensional ordered-subset expectation maximization (Fourier rebinning) method including corrections for scanner dead time and scattered radiation. Scans were analyzed for metabolic volume determination using eXplore VISTA (GE Healthcare). Metabolic uptake (in counts per pixel) in the pathologic and contralateral joint was determined from a volume of interest obtained by summing multiple 2-dimensional regions of interest from consecutive planes. At each time point and for each animal, joint metabolic ratio (JMR) was calculated as follows:

$$
\begin{aligned}
\mathrm{JMR}= & \text { metabolic uptake of pathologic paw } / \\
& \text { metabolic uptake of contralateral paw. }
\end{aligned}
$$

${ }^{99 m}$ Tc-HMDP Delayed Bone Phase Uptake Imaging. ${ }^{99 m}$ Tc-HMDP bone imaging was performed only to evaluate bony changes and not inflammation. Each animal underwent 3 examinations at days 4, 15, and 29. The delayed bone uptake phase was acquired $2 \mathrm{~h}$ after ${ }^{99 \mathrm{~m}} \mathrm{Tc}-$ HMDP injection (25 MBq/mouse; Osteocys [IBA]). Planar acquisition (10 $\mathrm{min}, 15 \%$ window at $140 \mathrm{keV}$ of ${ }^{99 \mathrm{~m}} \mathrm{Tc}$ ) was performed with animals positioned over a 10-cm collimator of a small-animal $\gamma$ camera ( $\gamma$ Imager; Biospace).

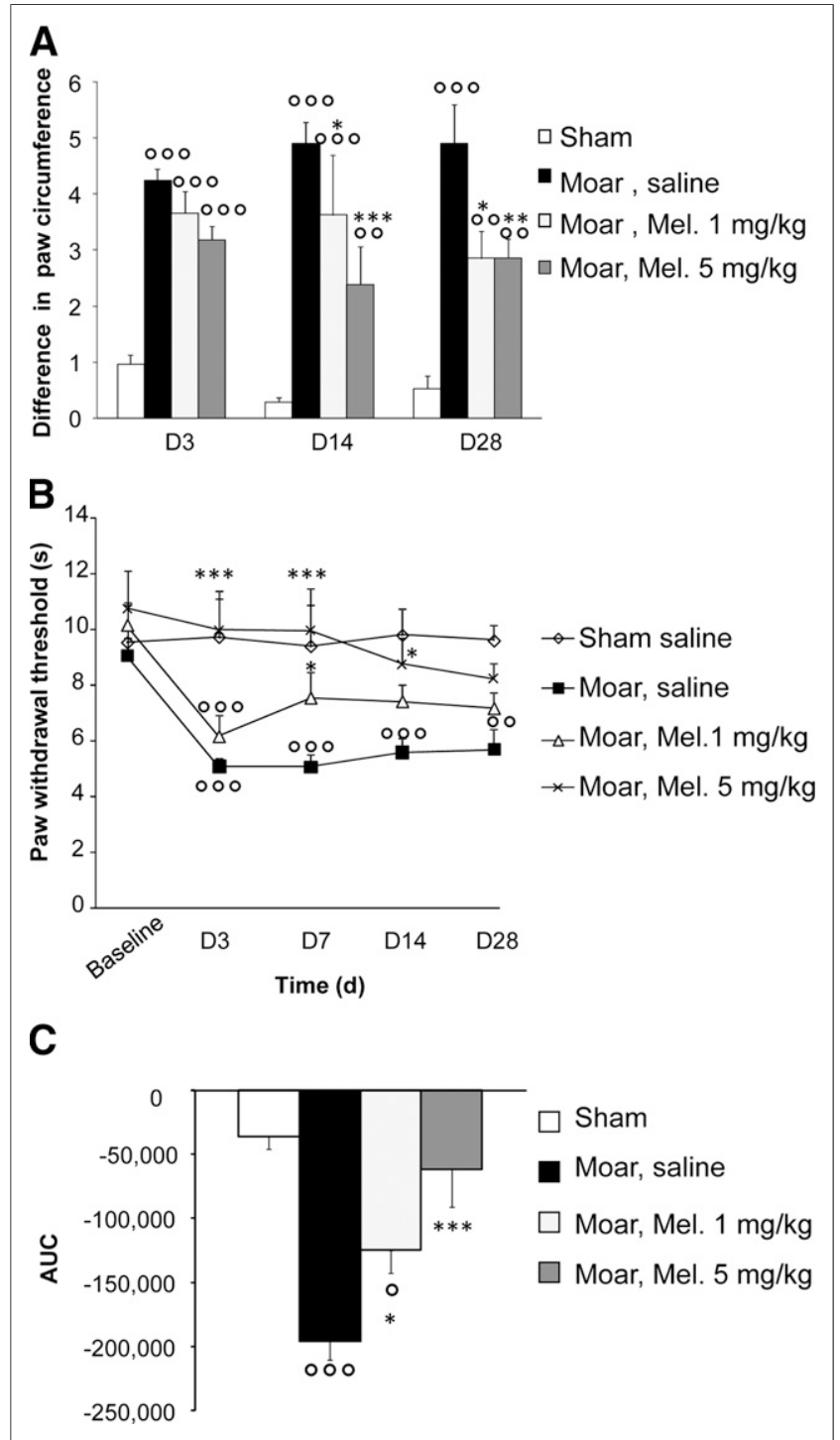

FIGURE 1. Clinical signs of monoarthritis, using paw circumference to measure edema $(A)$ and paw immersion test to assess thermal hyperalgesia (B) in shams, saline-treated Moars, or meloxicam-treated group (1 and $5 \mathrm{mg} / \mathrm{kg}$ ) at D3, D14, and D28. (C) AUC of meloxicam effect on thermal hyperalgesia in Moars is presented. Results are mean \pm SEM. ${ }^{\star} P<0.05,{ }^{\star \star} P<0.01,{ }^{\star \star \star} P<0.001$ vs. shams. ${ }^{\circ} P<0.05,{ }^{\circ} P<0.01$, ${ }^{\circ \circ} P<0.001$ vs. saline-treated Moars. $D=$ day; Mel $=$ meloxicam. 
Image processing was performed using Gammavision + (Biospace) with 2 identical fixed-sized rectangular regions of interest being delineated over the pathologic and contralateral joints.

At each time point and for each animal, the bone scintigraphic ratio $(\mathrm{SRb})$ was calculated as follows: $\mathrm{SR}_{\mathrm{b}}=$ average counts in pathologic paw/average counts in contralateral paw.

Cartilage Targeted Imaging Using ${ }^{99 m}$ Tc-NTP 15-5 Radiotracer. Each animal underwent 3 examinations at days 3, 14, and 28. Planar acquisition (10 $\mathrm{min}, 15 \%$ window at $140 \mathrm{keV}$ ) was performed $30 \mathrm{~min}$

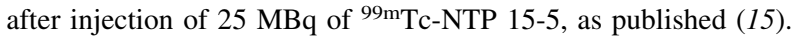

Quantitative analysis of scintigrams was performed with Gammavision + (Biospace), with fixed-size regions of interest delineated over the pathologic and contralateral joint, and total activity, average counts in cpm per pixel, and activity SD obtained. At each time point and for each animal, the cartilage scintigraphic ratio (SRc) was calculated as follows: $\mathrm{SRc}=$ average counts in pathologic joint/ average counts in contralateral joint.

\section{Proteoglycan Content of Whole-Joint Extract at Imaging Time Points and Histology}

At days 3,14, and 29, hind paw joints were removed ( $n=4-5$ animals per group) and crushed in liquid nitrogen. After incubation for $24 \mathrm{~h}$ at $60^{\circ} \mathrm{C}$ with ethylenediaminetetraacetic acid-phosphate buffer solution containing Papaine $(0.6 \mathrm{mg} / \mathrm{mL}$; Sigma-Aldrich) and DL-dithiothreitol
(DTT) $0.25 \mathrm{mg} / \mathrm{mL}$ (Sigma-Aldrich), digests were assessed for proteoglycan content using the dimethylene blue protocol (23).

At day 29, joints were removed and fixed during $48 \mathrm{~h}$ in $4 \%$ formaldehyde in phosphate buffer ( $0.1 \mathrm{M}, \mathrm{pH} 7.4)$ and then decalcified in $10 \%$ ethylenediaminetetraacetic acid (Sigma-Aldrich) during $2 \mathrm{wk}$ at $4^{\circ} \mathrm{C}$. After paraffin-embedding, 5- $\mu \mathrm{m}$ sections were stained with alcyan blue.

\section{Statistics}

Data are presented as mean \pm SEM. The significance level was a $P$ value of less than 0.05 .

A 2-way ANOVA was used to compare groups, followed by a StudentNewman-Keuls test (Sigma Stat 3.5) (Systat Software). To measure the global effect of meloxicam in the paw immersion test, areas under the time-course curve (AUCs) were calculated by the trapezoidal rule and expressed as mean \pm SEM (g.min). Variations of paw withdrawal latencies were calculated for each animal by the difference between latency at a given time and the corresponding control predrug (baseline) threshold. ANOVA followed by a Tukey test was used to analyze AUCs.

\section{RESULTS}

\section{Characterization of Monoarthritic Model}

A significant increase in paw circumference in Moars versus shams was observed at days 3,14, and 28. Meloxicam treatment significantly $(P<0.05)$ decreased paw circumference at days 14 and 28 versus saline but still stayed significantly different from the shams up to day 28 (Fig. 1A).

As expected, a significant decrease in paw withdrawal latency was observed in Moars at days 3, 7, 14, and 28 versus shams (Fig. 1B). Meloxicam increased paw withdrawal latency in a dose-dependent manner: when Moar animals were treated with the highest dose (5 mg/kg, subcutaneously), withdrawal latency values were similar to those observed for shams, for each time point investigated. AUC determination confirmed the dose-dependent response of paw withdrawal latency (Fig. 1C). However, when meloxicam was administered at 1 $\mathrm{mg} / \mathrm{kg}$, AUC was still significantly different from that of shams.
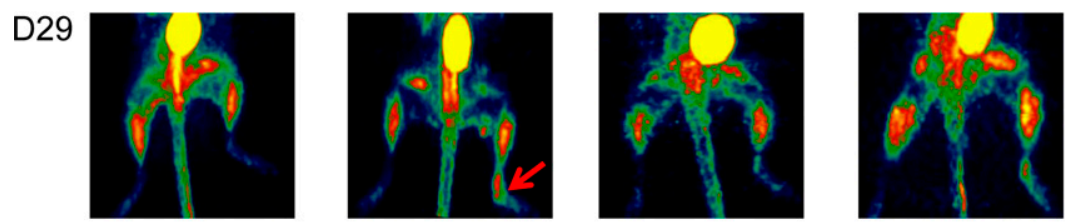

B

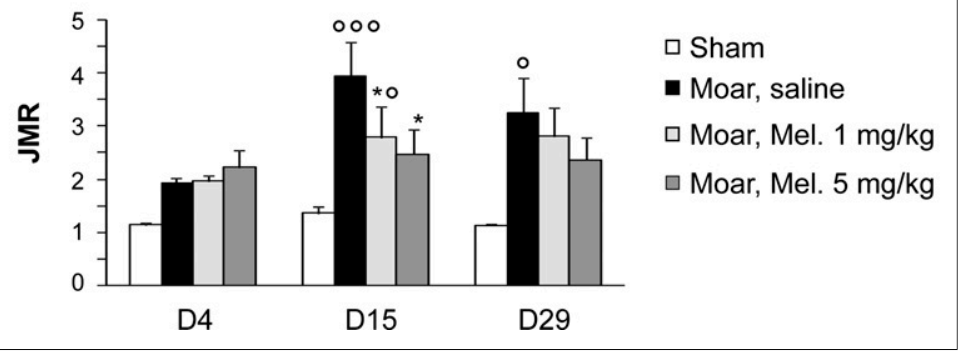

FIGURE 2. ${ }^{18} \mathrm{~F}-\mathrm{FDG}$ PET imaging. (A) Representative serial imaging in same animal of each group (i.e., sham, Moar + saline; Moar + meloxicam, $1 \mathrm{mg} / \mathrm{kg}$; Moar + meloxicam, $5 \mathrm{mg} / \mathrm{kg}$ ) at D4, D15, and D29. (B) Time course of JMR parameter (JMR = metabolic uptake of pathologic paw/metabolic uptake of contralateral paw). Results are mean \pm SEM. ${ }^{*} P<0.05$, ${ }^{* \star} P<0.01,{ }^{* \star *} P<0.001$ vs. shams. ${ }^{\circ} P<0.05,{ }^{\circ 0} P<0.01,{ }^{\circ 0 \circ} P<0.001$ vs. saline-treated Moar. $D=$ day; Mel = meloxicam.

\section{Functional Molecular Imaging}

${ }^{18}$ F-FDG Imaging of Inflammation. A higher ${ }^{18} \mathrm{~F}-\mathrm{FDG}$ accumulation was observed in Moars versus shams and was associated with a significant increase in JMR parameter at days 15 and $29(P<$ 0.05) (Fig. 2). Meloxicam at 1 and $5 \mathrm{mg} / \mathrm{kg}$ dose-dependently reduced ${ }^{18} \mathrm{~F}-\mathrm{FDG}$ accumulation, compared with saline-treated animals. Such decrease was statistically significant only at day 15 for both doses (Fig. 2B). Nevertheless, for Moar animals treated at $1 \mathrm{mg} / \mathrm{kg}$, JMR values were still significantly $(P<0.05)$ higher than those of shams.

${ }^{99 m}$ Tc-HMDP Delayed Bone Phase Uptake Imaging. An increased accumulation of ${ }^{99 m}$ Tc-HMDP was observed in Moars versus shams, with a significant increase 
of $\mathrm{SR}_{\mathrm{b}}(P<0.05)$ only at day 15 (Figs. 3A and 3B). At day 15, animals treated with both meloxicam doses exhibited a significant decrease $(P<0.05)$ in $\mathrm{SR}_{\mathrm{b}}$ versus saline-treated animals. However, the $\mathrm{SR}_{\mathrm{b}}$ of the $1 \mathrm{mg} / \mathrm{kg}$ group was still significantly $(P<$ $0.05)$ higher than that of shams. At day 29, no differences were observed in $\mathrm{SR}_{\mathrm{b}}$ between the different groups of animals.

${ }^{99 m}$ Tc-NTP 15-5 Cartilage Imaging. A differential accumulation of ${ }^{99 \mathrm{~m}} \mathrm{Tc}-\mathrm{NTP} 15-5$ occurred in the pathologic joint, respectively to the contralateral joint, in Moars at days 3, 14, and 28, with a significant $(P<0.05)$ increase in SRc versus shams (Fig. 4A). Meloxicam-treated mice $(5 \mathrm{mg} / \mathrm{kg})$ exhibited a significant $(P<0.05)$ decrease in Src versus saline-treated mice from day 14 (Fig. 4B).

To assess the in vivo specificity of ${ }^{99 \mathrm{~m}} \mathrm{Tc}-\mathrm{NTP} 15-5$ imaging and to exclude the possibility of unspecific uptake within the pathologic joint, additional Moars and shams $(n=4$ shams and $n=8$ Moars) were injected at day 14 with the nonvectorized equivalent of the radiotracer, named ${ }^{99 \mathrm{~m}} \mathrm{Tc}-15-5$ (formulae and scintigraphy obtained in a representative RA animal are given in Supplemental Fig. 1; supplemental materials are available at http://jnm.snmjournals. org): no significant difference was observed between Moars and shams, with mean $\mathrm{SRc}$ values of $0.97 \pm 0.15$ and $0.90 \pm 0.09$, respectively (Fig. 4C).

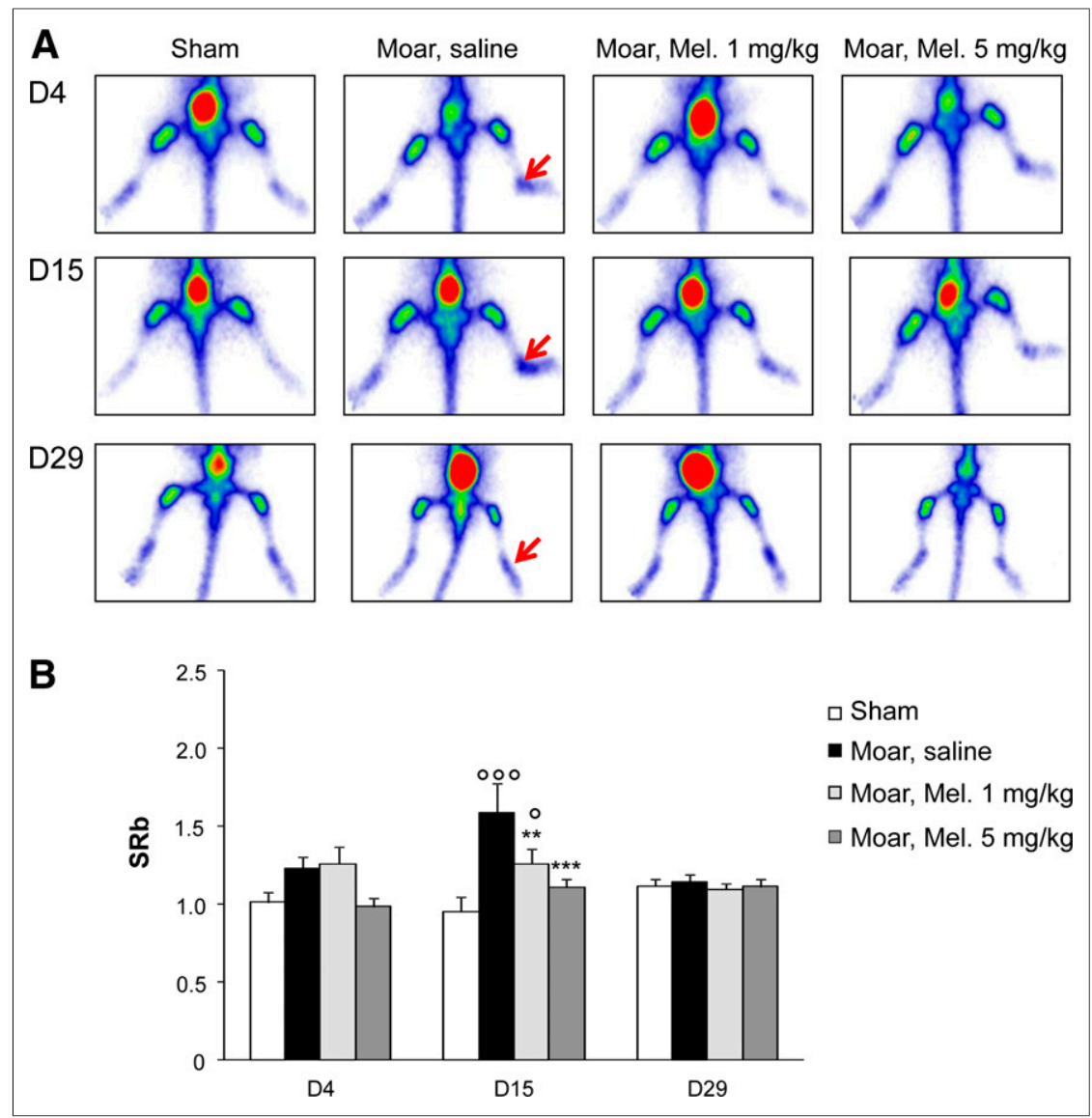

FIGURE 3. $\quad$ 99mTc-HMDP bone planar imaging. (A) Representative serial imaging in same animal of each group (i.e., sham, Moar + saline; Moar + meloxicam, $1 \mathrm{mg} / \mathrm{kg}$; Moar + meloxicam, $5 \mathrm{mg} / \mathrm{kg}$ ) at D4, D15, and D29. (B) Time course of $\mathrm{SR}_{\mathrm{b}}$ parameter $\left(\mathrm{SR}_{\mathrm{b}}=\right.$ average counts in pathologic paw/average counts contralateral paw). Results are mean \pm SEM. ${ }^{\star} P<0.05$, ${ }^{\star \star} P<0.01$, ${ }^{\star \star \star} P<$ 0.001 vs. shams. ${ }^{\circ} P<0.05,{ }^{\circ} P<0.01,{ }^{\circ 00} P<0.001$ vs. saline-treated Moar. $\mathrm{D}=$ day; Mel $=$ meloxicam.

\section{Histology of RA Joints at End of Study}

At day 29, histology (Fig. 5A) demonstrated a heterogeneous and decreased alcyan blue staining of the interarticular surface in Moars versus shams. When Moars were treated with meloxicam at $5 \mathrm{mg} / \mathrm{kg}$, alteration was not visualized.

\section{Proteoglycan Content of Whole-Joint Extract at Imaging Time Points}

To reinforce the results obtained with ${ }^{99 \mathrm{~m}} \mathrm{Tc}-\mathrm{NTP}$ 15-5 imaging, proteoglycan dosage of the whole-joint extracts was performed at days 3, 14, and 29: pathologic joints showed a significant $(P<$ 0.05 ) increase in joint proteoglycan content in Moars versus shams throughout the study (Fig. 5B).

For meloxicam groups, proteoglycan was reduced as compared with the saline-treated group at days 14 and 29, such as seen with ${ }^{99 m}$ Tc-NTP 15-5 imaging. At day 14, the highest reduction of proteoglycan content was observed for meloxicam at $5 \mathrm{mg} / \mathrm{kg}$.

\section{DISCUSSION}

99m Tc-NTP $15-5$ was previously demonstrated to be a candidate for the in vivo functional monitoring of proteoglycan remodeling associated with degenerative and tumoral pathologies of cartilage $(15-18,24)$. We evaluated, in an RA model, the relevance of $99 \mathrm{~m} \mathrm{Tc}-$ NTP 15-5 imaging for monitoring in vivo cartilage damage and response to nonsteroidal antiinflammatory drug therapy. Imaging parameters were studied in relation to the clinical signs and associated pain.

We chose the complete Freund adjuvant model in mice because it is characterized by lymphocyte infiltration into the synovium, increased joint volume, and cartilage destruction and led to functional alteration of the joint (25-27). This model is well known to present clinical changes, similar to stable chronic RA in human joints, such as synovitis, periarticular inflammation (paw swelling), histologic changes, thermal/ mechanical hypersensitivity, and importantly increased synovium proteoglycan content as indicating cartilage destruction. However, this model, helpful to identify early markers of the disease, is short lasting with a rapid development of inflammation and flare: it could be of interest to use a model with slower progression and affecting several joints.

In this model, we investigated the time course of cartilage remodeling by ${ }^{99 \mathrm{~m}} \mathrm{Tc}-\mathrm{NTP}$ $15-5$ scintigraphy, bone damages by ${ }^{99 \mathrm{~m}} \mathrm{Tc}$ HMDP imaging, inflammation by ${ }^{18}$ F-FDG PET, joint proteoglycan content, and pain behavior in animals, without and with meloxicam treatment, from day 1 to day 29 .

Using a paw immersion test, we observed thermal hypersensitivity in Moars as a significant decrease of thermal paw withdrawal latency up to day 28 as described (25-27). Our results confirmed that the complete Freund adjuvant model reproduced allodynia and hyperalgesia, as commonly described in 


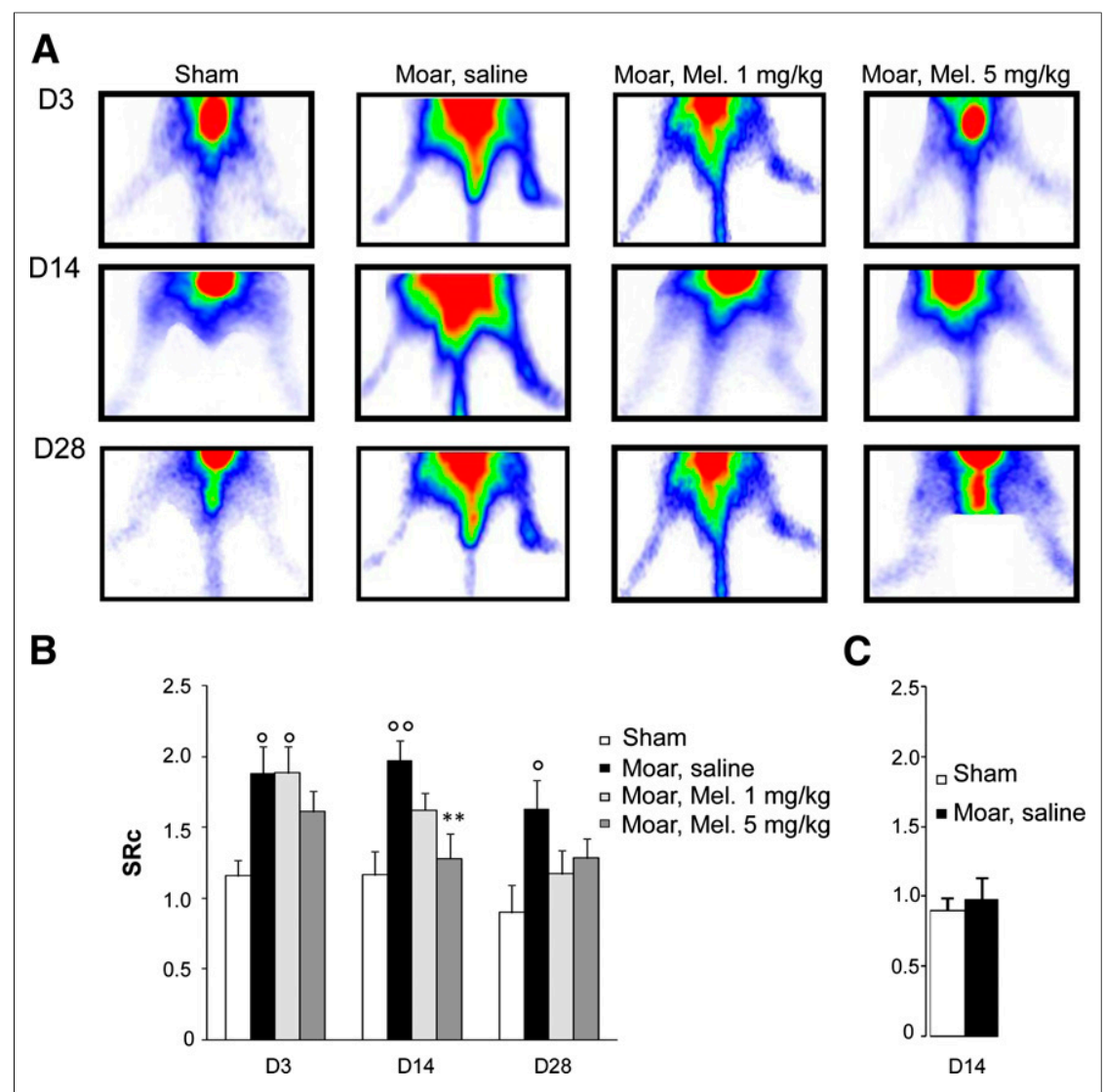

FIGURE 4. 99mTC-NTP 15-5 planar imaging. (A) Serial imaging in same animal of each group (sham, Moar + saline; Moar + meloxicam, $1 \mathrm{mg} / \mathrm{kg}$; Moar + meloxicam, $5 \mathrm{mg} / \mathrm{kg}$ ) at D4, D15, and D29. (B) Time course of SRc parameter calculated by average counts in pathologic paw/average counts contralateral paw. (C) SRc of shams and Moar injected with the nonvectorized $99 \mathrm{mTc}-15-5$ radiolabeled compound. Results are mean \pm SEM. ${ }^{*} P<0.05$, ${ }^{\star \star} P<0.01,{ }^{* \star *} P<0.001$ vs. shams. ${ }^{\circ} \mathrm{P}<0.05,{ }^{\circ 0} \mathrm{P}<0.01,{ }^{000} \mathrm{P}<0.001$ vs. saline-treated Moar. $\mathrm{D}=$ day; Mel $=$ meloxicam. this phenomenon was dose-dependently reduced by meloxicam. Such concordant results between ${ }^{99 \mathrm{~m}} \mathrm{Tc}-\mathrm{NTP} 15-5$ imaging and proteoglycan content of the whole RA joint reinforced the specificity of this radiotracer to assess cartilage remodeling and highlight its sensitivity to nonsteroidal antiinflammatory drugs, raising the question of the pathophysiologic significance of increased ${ }^{99 m}$ Tc-NTP $15-5$ accumulation in RA joints. Increased markers of cartilage turnover have been reported during the infraclinical course of RA in patients, suggesting that cartilage damage is an early feature. Moreover, disturbance of the cartilage matrix turnover is also well known to result in an increased release of its components, such as aggrecan fragments, into the synovial fluid. A compensatory matrix synthesis shift was also described with newly formed aggrecan components but not incorporated in joint tissues (32-35). In addition, Brescia et al. highlighted the role of fibroblast-like synoviocytes as an important cell population in the synovium of RA patients, highly expressing aggrecan messenger RNA level (2.91 fold higher vs. controls) and exhibiting cartilage-like phenotype (36).

The ${ }^{99 m}$ Tc-NTP 15-5 SRc parameter in monoarthritic joints could be considered to reflect all multifactorial physiopathologic and biochemical changes at the whole-joint in vivo level, such as proteoglycan interactions and fibroblast-like synoviocyte phenotype in synovial fluid as well as proteoglycan turnover in cartilage tissue. The

patients (28). Pain behavior was dose-dependently reduced by meloxicam as previously shown in inflammatory pain models $(21,29-30)$. Signs of inflammation were evidenced as paw circumference (i.e., edema) increase in Moars from day 3 to day 28. As expected, an increased accumulation of ${ }^{18} \mathrm{~F}$-FDG in the RA joint was observed and attenuated by meloxicam. The pathophysiologic significance of ${ }^{18} \mathrm{~F}$ FDG accumulation in joints with RA is considered to reflect both the early infiltrating inflammatory cells and the late proliferating fibroblasts in pannus (31). Histology at day 29 confirmed loss and heterogeneous alcyan blue staining at the interarticular surface indicative of cartilage erosion, which was reduced by meloxicam at $5 \mathrm{mg} / \mathrm{kg}$.

The cartilage integrity of the RA joint was monitored in vivo by serial ${ }^{99 m}$ Tc-NTP 15-5 imaging. A time-dependant increase in SRc uptake ratio was observed in Moars versus shams. Increased accumulation of ${ }^{99 m}$ Tc-NTP $15-5$ in Moar joints was dose-dependently reduced by meloxicam.

To test the hypothesis of unspecific uptake of ${ }^{99 m}$ Tc-NTP 15-5 attributable to inflammation in RA joints, Moars and shams were injected with the nonvectorized radiotracer, ${ }^{99 \mathrm{~m}} \mathrm{Tc}-15-5$ : no differences in joint uptake were observed between Moars and shams, with SRc values around 1 for both groups.

${ }^{99 m}$ Tc-NTP 15-5 uptake was compared with proteoglycan content of the whole RA joints. An increased proteoglycan content in Moars was observed versus shams over the study; interestingly, mechanism at its origin needs to be further elucidated.

Interestingly, the ${ }^{99 \mathrm{~m}} \mathrm{Tc}-\mathrm{NTP} 15-5 \mathrm{SRc}$ parameter and joint proteoglycan content were decreased by meloxicam: such results are in agreement with literature, which found meloxicam to have a beneficial effect in vitro on the overall proteoglycan metabolism of cartilage explants from patients (37).

Because RA is also associated with bone damage, animals were submitted to ${ }^{99 m} \mathrm{Tc}-\mathrm{HMDP}$ imaging. No difference in ${ }^{99 \mathrm{~m}} \mathrm{Tc}-$ HMDP uptake was observed between the early and late stage of the pathology. Intriguingly, an increase in the $\mathrm{SR}_{\mathrm{b}}$ ratio, reflecting bone remodeling, was observed only at day 15 in Moars versus shams and was dose-dependently reduced by meloxicam. Bone remodeling is mainly recognized as occurring secondary to the loss of cartilage, with subchondral osteolysis developing in the late disease (38).

Several innovative approaches have been already explored for molecular imaging of RA, including probes targeting cell proliferation, matrix metalloproteinases, folate receptors, macrophage mannose receptor with antibodies, or nanobodies, with the key of

In this model, the early joint imaging patterns evidenced by the ${ }^{99 \mathrm{~m}}$ Tc-NTP 15-5 radiotracer as compared with ${ }^{99 \mathrm{~m}} \mathrm{Tc}-\mathrm{HMDP}$ and ${ }^{18}$ F-FDG conventionally used highlight its high potential for RA. ${ }^{99 m}$ Tc-NTP $15-5$ imaging may be useful to assess cartilage success depending of the sensitivity of the markers (39-42). 


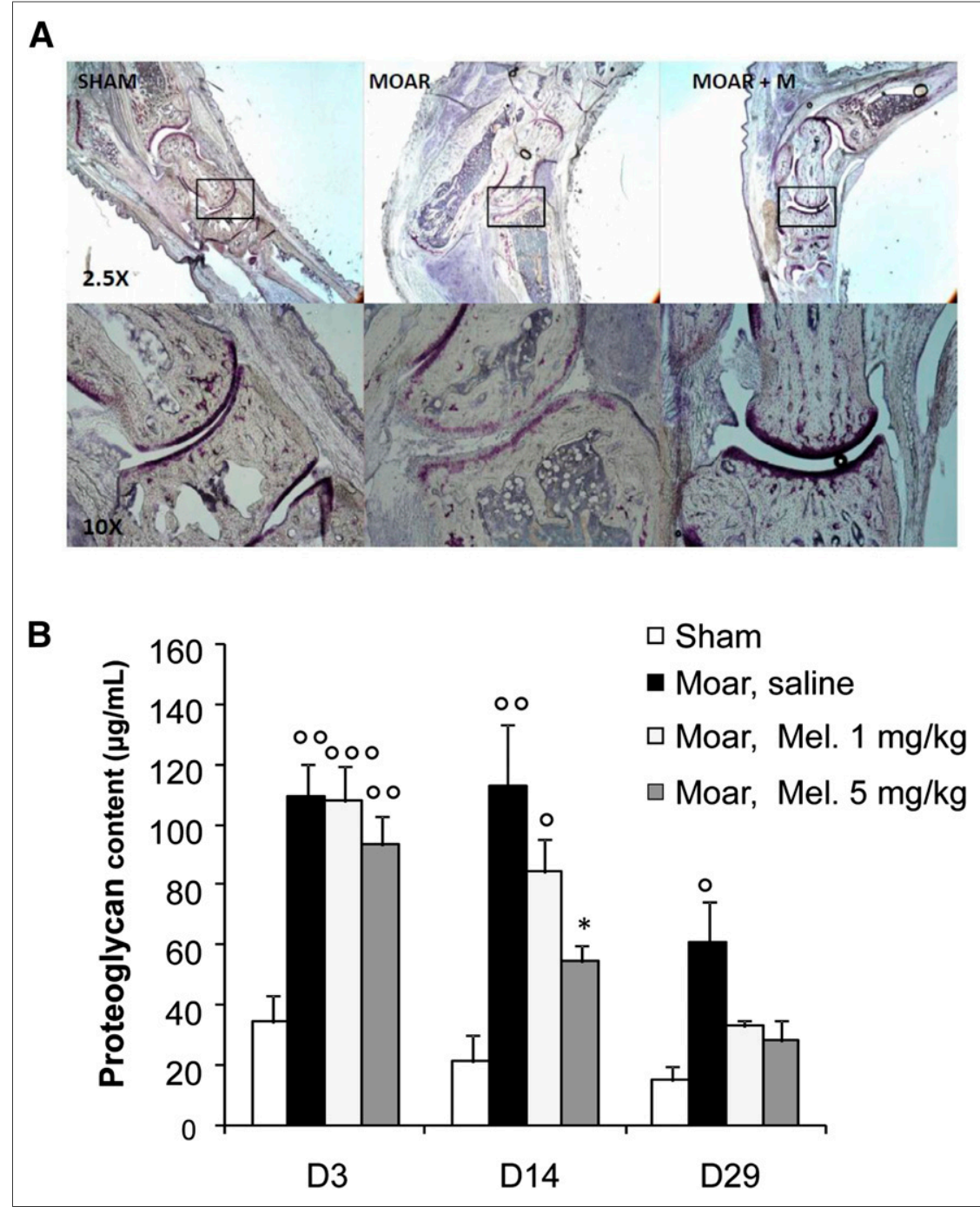

FIGURE 5. Joint analysis. (A) Alcyan blue staining of pathologic joints of shams, Moar + saline, and Moar + meloxicam, $5 \mathrm{mg} / \mathrm{kg}$, at D29. (B) Proteoglycan content of whole joints using dimethylmethylene blue assay at D3, D14, and D29 ( $n=4-5$ animals per group). Results are mean \pm SEM. ${ }^{\star} P<0.05,{ }^{\star \star} P<0.01,{ }^{\star \star \star} P<0.001$ vs. sham. ${ }^{\circ} P<0.05,{ }^{\circ} P<0.01,{ }^{\circ 00}: P<0.001$ vs. saline-treated Moar. $\mathrm{D}=$ day; Mel = meloxicam. drugs demonstrated in this paper may offer the opportunity to provide an in vivo suitable set of criteria to quantify cartilage integrity and functionality, for assessing new emerging disease-modifying antirheumatic drugs strategies.

In clinics, the major challenge of ${ }^{99 \mathrm{~m}} \mathrm{Tc}-$ NTP 15-5 imaging will be obtaining sufficient scintigraphic sensitivity to correlate tracer uptake and proteoglycan content, to evaluate small changes at earlier stages.

\section{CONCLUSION}

${ }^{99 m}$ Tc-NTP 15-5 imaging is not intended to be the sole molecular imaging technique for diagnosing RA but rather to be used for assessing cartilage damage in RA. Considering that the ${ }^{99 \mathrm{~m}} \mathrm{Tc}-\mathrm{NTP} 15-5$ radiotracer is currently under validation for clinical use in humans, this may represent the opportunity to bridge the gap between preclinical and clinical testing in RA.

\section{DISCLOSURE}

The costs of publication of this article were defrayed in part by the payment of page charges. Therefore, and solely to indicate this fact, this article is hereby marked "advertisement" in accordance with 18 USC section 1734. No potential conflict of interest relevant to this article was reported.

\section{ACKNOWLEDGMENTS}

Region Auvergne, Innovation, and Auvergne Regional Work was conducted in collaboration with Cyclopharma Laboratoires. Dr. Khairnar acknowledges the funding received from the foundation of Auvergne University from 2011 to 2012. involvement and remodeling in the pathology, in addition to inflammation imaging with ${ }^{18} \mathrm{~F}-\mathrm{FDG}$, and also bone component with 99m Tc-HMDP.

For the evaluation of cartilage proteoglycans, others biophysical methods have been described: high-frequency ultrasound provides information about proteoglycan content in cartilage specimens in vitro but seems difficult to apply in vivo (43). Gadoliniumdiethylenetriaminepentaacetic acid-enhanced MR imaging offers an estimate of the local concentration of the contrast agent, which accumulates in cartilage inversely to the negative charges of glycosaminoglycans (13). This method allows only an indirect measurement of proteoglycan content and requires a high dose of contrast agent (and nonnegligible associated toxicity) and is known to be several orders of magnitude less sensitive than nuclear imaging.

In light of these encouraging results, the sensitivity of ${ }^{99 \mathrm{~m}} \mathrm{Tc}-$ NTP 15-5 imaging should be assessed for characterizing a model of slower disease progression that affects several joints. The sensitivity of ${ }^{99 \mathrm{~m}} \mathrm{Tc}-\mathrm{NTP} 15-5$ imaging to nonsteroidal antiinflammatory

\section{REFERENCES}

1. Strand V, Khanna D. The impact of rheumatoid arthritis and treatment on patients' lives. Clin Exp Rheumatol. 2010;28:S32-S40.

2. Boonen A, Severens JL. The burden of illness of rheumatoid arthritis. Clin Rheumatol. 2011;30(suppl 1):S3-S8.

3. Walsh DA, McWilliams DF. Mechanisms, impact and management of pain in rheumatoid arthritis. Nat Rev Rheumatol. 2014;10:581-592.

4. Haro I, Sanmartí R. Rheumatoid arthritis: current advances in pathogenesis, diagnosis and therapy. Curr Top Med Chem. 2013;13:697.

5. Sanmartí R, Ruiz-Esquide V, Hernández MV. Rheumatoid arthritis: a clinical overview of new diagnostic and treatment approaches. Curr Top Med Chem. 2013;13:698-704.

6. Zeidler H. The need to better classify and diagnose early and very early rheumatoid arthritis. J Rheumatol. 2012;39:212-217.

7. Nam JL, Ramiro S, Gaujoux-Viala C, et al. Efficacy of biological diseasemodifying antirheumatic drugs: a systematic literature review informing the 2013 update of the EULAR recommendations for the management of rheumatoid arthritis. Ann Rheum Dis. 2014;73:516-528.

8. Gompels LL, Paleolog EM. A window on disease pathogenesis and potential therapeutic strategies: molecular imaging for arthritis. Arthritis Res Ther. 2011;13:201-213.

9. McQueen FM. Imaging in early rheumatoid arthritis. Best Pract Res Clin Rheumatol. 2013;27:499-522. 
10. Aletaha D, Funovits J, Smolen JS. Physical disability in rheumatoid arthritis is associated with cartilage damage rather than bone destruction. Ann Rheum Dis. 2011;70:733-739.

11. Korb-Pap A, Stratis A, Mühlenberg K, et al. Early structural changes in cartilage and bone are required for the attachment and invasion of inflamed synovial tissue during destructive inflammatory arthritis. Ann Rheum Dis. 2012;71:1004-1011.

12. Frisenda S, Perricone C, Valesini G. Cartilage as a target of autoimmunity: a review. Autoimmun Rev. 2013;12:591-598.

13. Miese F, Buchbender C, Scherer A, et al. Molecular imaging of cartilage damage of finger joints in early rheumatoid arthritis with delayed gadolinium-enhanced magnetic resonance imaging. Arthritis Rheum. 2012;64:394-399.

14. Maurizis JC, Rapp M, Nicolas C, et al. Disposition in rats of $N$-pyridinium-propylcyclam, $N$-triethy-lammonium-propyl-cyclam, and $N$-[triethylammonium]-3-propyl[15]ane-N5, potential cartilage imaging agents. Drug Metab Dispos. 2000;28:418-422.

15. Miot-Noirault E, Vidal A, Pastoureau P, et al. Early detection and monitoring of cartilage alteration in the experimental meniscectomised guinea pig model of osteoarthritis by ${ }^{99 \mathrm{~m}}$ Tc-NTP 15-5 scintigraphy. Eur J Nucl Med Mol Imaging. 2007;34:1280-1290.

16. Miot-Noirault E, Vidal A, Auzeloux $\mathrm{P}$, et al. In vivo scintigraphic imaging of proteoglycans. Methods Mol Biol. 2012;836:183-198.

17. Cachin F, Boisgard S, Vidal A, et al. First ex vivo study demonstrating that 99mTc-NTP 15-5 radiotracer binds to human articular cartilage. Eur J Nucl Med Mol Imaging. 2011;38:2077-2082.

18. Miot-Noirault E, Guicheux J, Vidal A, et al. In vivo experimental imaging of osteochondral defects and their healing using ${ }^{99 \mathrm{~m}} \mathrm{Tc}-\mathrm{NTP} 15-5$ radiotracer. Eur $J$ Nucl Med Mol Imaging. 2012;39:1169-1172.

19. Butler SH, Godefroy F, Besson JM, Weil-Fugazza J. A limited arthritic model for chronic pain studies in the rat. Pain. 1992;48:73-81.

20. Torres-Guzman AM, Morado-Urbina CE, Alvarado-Vazquez PA, et al. Chronic oral or intraarticular administration of docosahexaenoic acid reduces nociception and knee edema and improves functional outcomes in a mouse model of complete Freund's adjuvant-induced knee arthritis. Arthritis Res Ther. 2014;16:R64R75.

21. Hashmi JA, Yashpal K, Holdsworth DW, Henry JL. Sensory and vascular changes in a rat monoarthritis model: prophylactic and therapeutic effects of meloxicam. Inflamm Res. 2010;59:667-678.

22. Laird JM, Herrero JF, García de la Rubia P, Cervero F. Analgesic activity of the novel COX-2 preferring NSAID, meloxicam in mono-arthritic rats: central and peripheral components. Inflamm Res. 1997;46:203-210.

23. Farndale RW, Buttle DJ, Barrett AJ. Improved quantitation and discrimination of sulphated glycosaminoglycans by use of dimethylmethylene blue. Biochim Biophys Acta. 1986;883:173-177.

24. Miot-Noirault E, Gouin F, Vidal A, et al. First preclinical imaging of primary cartilage neoplasm and its local recurrence using ${ }^{99 \mathrm{~m}} \mathrm{Tc}-\mathrm{NTP} 15-5$ radiotracer. J Nucl Med. 2009;50:1541-1547.

25. Hu Y, Cheng W, Cai W, Yue Y, Li J, Zhang P. Advances in research on animal models of rheumatoid arthritis. Clin Rheumatol. 2013;32:161-165.

26. Millecamps M, Jourdan D, Leger S, et al. Circadian pattern of spontaneous behavior in monarthritic rats: a novel global approach to evaluation of chronic pain and treatment effectiveness. Arthritis Rheum. 2005;52:3470-3478.
27. Koo ST, Lee CH, Choi $\mathrm{H}$, et al. The effects of pressure on arthritic knees in a rat model of CFA-induced arthritis. Pain Physician. 2013;16:E95-E102.

28. Meeus M, Vervisch S, De Clerck LS, Moorkens G, Hans G, Nijs J. Central sensitization in patients with rheumatoid arthritis: a systematic literature review. Semin Arthritis Rheum. 2012;41:556-567.

29. Grégoire S, Wattiez AS, Etienne M, et al. Monoarthritis-induced emotional and cognitive impairments in rats are sensitive to low systemic doses or intra-amygdala injections of morphine. Eur J Pharmacol. 2014;735:1-9.

30. Ahmed M, Khanna D, Furst DE. Meloxicam in rheumatoid arthritis. Expert Opin Drug Metab Toxicol. 2005;1:739-751.

31. Mountz JM, Alavi A, Mountz D. Emerging optical and nuclear medicine imaging methods in rheumatoid arthritis. Nat Rev Rheum. 2012;8:719-728.

32. Fraser A, Fearon U, Billinghurst RC, et al. Turnover of type II collagen and aggrecan in cartilage matrix at the onset of inflammatory arthritis in humans: relationship to mediators of systemic and local inflammation. Arthritis Rheum. 2003;48:3085-3095.

33. Ishiguro $\mathrm{N}$, Ito $\mathrm{T}$, Ito $\mathrm{H}$, et al. Relationship of matrix metalloproteinases and their inhibitors to cartilage proteoglycan and collagen turnover. Arthritis Rheum. 1999;42:129-136.

34. Turesson C, Bergström U, Jacobsson LT, et al. Increased cartilage turnover and circulating autoanti-bodies in different subsets before the clinical onset of rheumatoid arthritis. Ann Rheum Dis. 2011;70:520-522.

35. Struglics A, Hansson M, Lohmander LS. Human aggrecanase generated synovial fluid fragment levels are elevated directly after knee injuries due to proteolysis both in the inter globular and chondroitin sulfate domains. Osteoarthritis Cartilage. 2011;19:1047-1057.

36. Brescia AC, Simonds MM, Mc Cahan M, Fawcett PT, Rose CD. The role of transforming growth factor $\beta$ signaling in fibroblast-like synoviocytes from patients with oligoarticular juvenile idiopathic arthritis. Arthritis Rheumatol. 2014;66:1352-1362.

37. Blot L, Marcelis A, Devogelaer JP, Manicourt DH. Effects of diclofenac, aceclofenac and meloxicam on the metabolism of proteoglycans and hyaluronan in osteoarthritic human cartilage. Br J Pharmacol. 2000;131:1413-1421.

38. Deal C. Bone loss in rheumatoid arthritis: systemic, periarticular, and focal. Curr Rheumatol Rep. 2012;14:231-237.

39. Fuchs K, Kohlhofer U, Quintanilla-Martinez L, et al. In vivo imaging of cell proliferation enables the detection of the extent of experimental rheumatoid arthritis by $3^{\prime}$-deoxy-3'-18f-fluorothymidine and small-animal PET. J Nucl Med. 2013;54:151-158.

40. Ryu JH, Lee A, Chu JU, et al. Early diagnosis of arthritis in mice with collageninduced arthritis, using a fluorogenic matrix metalloproteinase 3-specific polymeric probe. Arthritis Rheum. 2011;63:3824-3832.

41. Piscaer TM, Müller C, Mindt TL, et al. Imaging of activated macrophages in experimental osteoarthritis using folate-targeted animal single-photon-emission computed tomography/computed tomography. Arthritis Rheum. 2011;63:1898-1907.

42. Put S, Schoonooghe S, Devoogdt N, et al. SPECT imaging of joint inflammation with Nanobodies targeting the macrophage mannose receptor in a mouse model for rheumatoid arthritis. J Nucl Med. 2013;54:807-814.

43. Wang Q, Zheng YP, Qin L, et al. Real-time ultrasonic assessment of progressive proteoglycan depletion in articular cartilage. Ultrasound Med Biol. 2008;34:1085-1092. 